\title{
Forschung, Praxis und Lehre vernetzen
}

\section{Das Kompetenzzentrum für Soziale Arbeit in Wien}

\author{
Christine Gruber
}

FH-Prof. Mag. Dr. Christine Gruber ist Studiengangsleiterin, Leiterin des Erasmus-Projekts »SOWOSEC - Soziale Arbeit und Sozialwirtschaft « und Leiterin des Kompetenzzentrums für Soziale Arbeit (KOSAR) an der FH Campus Wien.

E-Mail

christine.gruber@fh-campuswien.ac.at
Das Kompetenzzentrum für Soziale Arbeit an der Fachbochschule Wien ist angesiedelt an der Schnittstelle von Forschung, Praxis und Lehre. Ziel der Einrichtung ist es, das Wissens- und Innovationspotenzial aus diesen drei Bereichen an einem Ort zusammenzuführen und die gewonnenen Erkenntnisse maßgeschneidert für unterschiedliche Projekte nutzbar zu machen. Das Wiener Kompetenzzentrum ist damit ein Beispiel für die in den letzten Jahren entstandenen neuen Modelle der träger-, arbeitsfeld- und professionsübergreifenden Kooperation in der Sozialen Arbeit.

Das Kompetenzzentrum für Soziale Arbeit (KOSAR) wurde 2005 als rechtlich eigenständige Tochtergesellschaft des FHTrägervereins »FH Campus Wien - Verein zur Förderung des Fachhochschul-, Entwicklungs- und Forschungszentrums im Süden Wiens « gegründet und versteht sich primär als Forschungseinrichtung für Fragestellungen der Sozialen Arbeit und Sozialwirtschaft. Die Organisation ist an der Schnittstelle von Forschung, Praxis und Lehre in der Sozialen Arbeit angesiedelt und führt das Wissens- und Innovationspotenzial aus diesen drei Bereichen an einem Ort zusammen.

Das Angebotsprofil des Kompetenzzentrums für Soziale Arbeit richtet sich inhaltlich an zentralen Problemstellungen und Themen der Sozialen Arbeit aus und orientiert sich an den Bedarfslagen der sozialen Berufspraxis, der Sozialwirtschaft sowie von Politik und Verwaltung. Alle Vorhaben werden auf ihre Relevanz für die Theorieentwicklung sowie Praxis der Sozialen Arbeit, Sozialwirtschaft und Sozialpolitik hin geprüft. Die Angebote umfassen:

- Forschung und Entwicklung (Schwerpunkt der Aktivitäten des Kompetenzzentrums für Soziale Arbeit)

- wissenschaftliche Beratung und Expertise

- Fort- und Weiterbildung
Die Aktivitäten in Forschung und Entwicklung des Kompetenzzentrums setzen an einem in Österreich bislang weitgehend vernachlässigten Bedarf an Innovation und Forschung für die Praxis der Sozialen Arbeit und Sozialwirtschaft an, der national und international von steigender Bedeutung ist. Sie stehen im Zeichen der dringend nötigen Stärkung und Etablierung der in Österreich bisher strukturell nur sehr unzureichend verankerten Sozialarbeitsforschung. Mit innovativer Forschung und Entwicklung in diesem Feld kann und soll ein essenzieller Beitrag zur zukunftsweisenden Weiterentwicklung des Gesellschaftsbereichs Soziale Arbeit und damit zugleich zur gesellschaftlichen Entwicklung insgesamt geleistet werden.

\section{Forschung}

Die Forschung ist das Kerngeschäft des Kompetenzzentrums für Soziale Arbeit und als anwendungsorientierte empirische Sozialforschung im Bereich der Sozialen Arbeit konzipiert. Die Produkte sind dienstleistungsorientiert und erarbeiten Planungs- und Entscheidungsgrundlagen für private und öffentliche soziale Einrichtungen sowie für die Sozialpolitik. Alle Projekte generieren theoriegeleitetes, umsetzungs- und handlungsrelevantes Wissen und sichern die Entwicklung neuer Instrumente für die Soziale Arbeit. Kooperationen mit Hochschul- sowie Forschungseinrichtungen und Praxisinstitutionen sind teilweise bereits realisiert und werden weiter ausgebaut.

Die strategische Planung und Positionierung des Kompetenzzentrums für Soziale Arbeit als Forschungs- und Entwicklungs-Tochtergesellschaft der FH Campus Wien beinhaltet als Kernaufgaben:

- die laufende Konzeptentwicklung und Projektakquisition sowie

- das Projektmanagement und die wissenschaftliche Durchführung von sozi- 
alarbeiterisch relevanten Forschungsund Entwicklungsprojekten

- die Netzwerkarbeit in der Fachöffentlichkeit und den Wissenstransfer in Richtung Ausbildung, Berufspraxis, Scientific Community, Sozialwirtschaft, Politik und Gesellschaft.

Die Forschungsfelder und Forschungsschwerpunkte des Kompetenzzentrums für Soziale Arbeit sind den drei Erfolgsbereichen "Lebensbedingungen «, "Sicherheiten « und »Berufswelten und Qualifikationen « zugeordnet (vgl. Grafik), an denen sich alle eigenständigen Forschungs- und Entwicklungseinrichtungen in den unterschiedlichen Fachbereichen an der FH Campus Wien ausrichten.
In der Entwicklung und Durchführung der Forschungs- und Entwicklungsprojekte am Kompetenzzentrum für Soziale Arbeit wird quer zu allen Forschungsfel- von Projekten im Sozialbereich. Durch Projektbegleitung und Evaluierung wird es möglich, sozialarbeiterisches Handeln in der Praxis wissenschaftlich zu unter-

\section{»Entscheidend ist der Transfer in Lebre und Praxis"}

dern und Schwerpunkten die Gender-Perspektive berücksichtigt. Ein besonderer Schwerpunkt des Kompetenzzentrums für Soziale Arbeit liegt in der wissenschaftlichen Begleitung und Evaluierung stützen. Sozialarbeitsforschung wird so zu einer wichtigen Qualitätssicherungsmaßnahme für die soziale Praxis.

Bei der Abwicklung der Projekte und Aufträge kann das Kompetenzzentrum

Das Kompetenzzentrum für Soziale Arbeit an der Fachhochschule Wien will in Projekten Forschung, Praxis und Lehre zusammenführen, wie einige Beispiele zeigen können:

Projekt: Evaluierung sozialer Interventionen

Inhalt: Ziel ist es einerseits, durch die Entwicklung und Anwendung von Evaluationsansätzen in der Sozialarbeit die Theoriebildung über die Wirkung sozialer Interventionen für ein ausgewähltes Praxisfeld zu fördern; andererseits soll die Interventionspraxis durch die Entwicklung von Maßnahmenkonzepten fundiert werden.

Projekt: Subjektive Wahrnehmung von Sicherheit und Unsicherheit im öffentlichen Raum

Inhalt: Das Projekt möchte einen Beitrag dazu leisten, das Wissen über den subjektiven Definitionsprozess von »Sicherheit « und »Unsicherheit « im öffentlichen Raum zu erhöhen. Untersucht werden unterschiedliche Nutzergruppen sowie Organisationen im sozialen Bereich, die in diesen Räumen tätig sind (Sozialarbeiterinnen und Sozialarbeiter, Polizistinnen und Polizisten, Security-Mitarbeiterinnen und Security-Mitarbeiter, Geschäftstreibende etc.). Ziel ist ein besseres Verständnis des subjektiven Sicherheits-Wahrnehmungsprozesses unterschiedlicher Bevölkerungsgruppen sowie die Entwicklung von Schulungsmaterial für interessierte - im öffentlichen Raum tätige - Institutionen (Exekutive, Soziale Arbeit etc.).

Projekt: Glossar »Soziale Arbeit im (teil)öffentlichen Raum« Inhalt: Erstellung eines Glossars mit Begriffen und Bezügen, die bei der Sozialen Arbeit im (teil)öffentlichen Raum in Wien verwendet werden. Parallel zum empirischen Zugang des Begriffsgebrauchs wird eine theoretische Klärung der Begriffe Grundlage des Glossars sein. Betrachtet werden Einrichtungen, die folgenden Geschäftsgruppen der Stadt Wien zugeordnet sind: Bildung, Jugend, Information und Sport; Gesundheit und Soziales Integration, Frauenfragen, Konsumentenschutz und Personal; Stadtentwicklung und Verkehr; Wohnen, Wohnbau und Stadterneuerung. Über eine Rükkkoppelungsschleife (Workshops, Gruppeninterviews) wird die betroffene Praxis (v. a. auf Auftragnehmer-Ebene) einbezogen.

\section{Projekt: Sozialraumanalyse im öffentlichen Raum}

Inhalt: Im Rahmen einer Kooperation mit der Planungsfirma "tilia « wird in einem öffentlichen Raum eine modellhafte Sozialraumanalyse entwickelt und durchgeführt. Dabei sollen nicht nur die Interessen (potenzieller) Nutzerinnen und Nutzer in die Planung einfließen können, sondern auch ein Leitfaden erstellt werden für zukünftige Sozialraumanalysen im öffentlichen Raum.

Projekt: Schulsozialarbeit an Grundschulen; Strukturevaluation eines Modellprojektes an einer Wiener Volksschule Inhalt: Ziel ist, das Modellprojekt hinsichtlich der Implementierungsbedingungen und das Interventionskonzept "NEUSTART - voll fair « hinsichtlich der inhaltlich-fachlichen Zielsetzungen zu bewerten. Als Bewertungskriterien werden fachliche Qualitätsstandards für Schulsozialarbeit im Grundschulbereich herangezogen. Inhaltlich setzt dabei die Evaluation auf Struktur- und Konzeptebene an.

Projekt: Betriebliche Frauenförderung

Inhalt: Die Studie im Auftrag des Frauenbüros der Stadt Wien setzt sich mit betrieblicher Frauenförderung auseinander.

Projekt: Begleitstudie zum Pilotprojekt persönliche Assistenz Inhalt: Die Studie im Auftrag des Fonds Soziales Wien dient dazu, Erfahrungen mit dem Leistungsangebot persönliche Assistenz zu sammeln, Potentiale und Risiken zu analysieren und ein für Wien optimiertes Modell der Persönlichen Assistenz zu entwickeln.

Weitere Informationen bietet die Website des Kompetenzzentrums für Soziale Arbeit an der FH Campus Wien: http://www.fh-campuswien.ac.at/kosar. 
für Soziale Arbeit auf die Know-howRessourcen der forschungs- und praxiserfahrenen Lehrenden der FH-Studiengänge des Departments Soziales an der Fachhochschule Wien zurückgreifen. Dadurch steht der Forschungseinrichtung ein einzigartiger Wissens- und Erfahrungspool zur Verfügung, der ergänzt wird durch den Auf- und Ausbau eines breiten und fundierten Forschungs-Know-hows der wissenschaftlichen Mitarbeiterinnen und Mitarbeitern des Kompetenzzentrums für Soziale Arbeit. Die Projektteams spiegeln die Kombination aus Forschungs- und sozialarbeiterischer Kompetenz wider und bilden die Grundlage für die besondere Qualität der Forschungen des Kompetenzzentrums. Für sämtliche in der For- schung und Entwicklung gewonnenen Erkenntnisse wird ein umfassender Wissenstransfer in die Lehre und Praxis Sozialer Arbeit angestrebt.

Die Ziele der Forschungseinrichtung lassen sich wie folgt zusammenfassen:

- Etablierung der Sozialarbeitsforschung in Österreich als essenziellen Beitrag zur weiteren Professionalisierung des Berufsfeldes, zur innovativen Weiterentwicklung des Gesellschaftsbereichs Soziale Arbeit und zur gesamtgesellschaftlichen Kohäsion

- Entwicklung neuer Instrumente und Lösungswege für die Soziale Arbeit und Generierung wissenschaftlich-empirisch gesicherter Planungs- und Entscheidungsgrundlagen für Sozialpoliti-

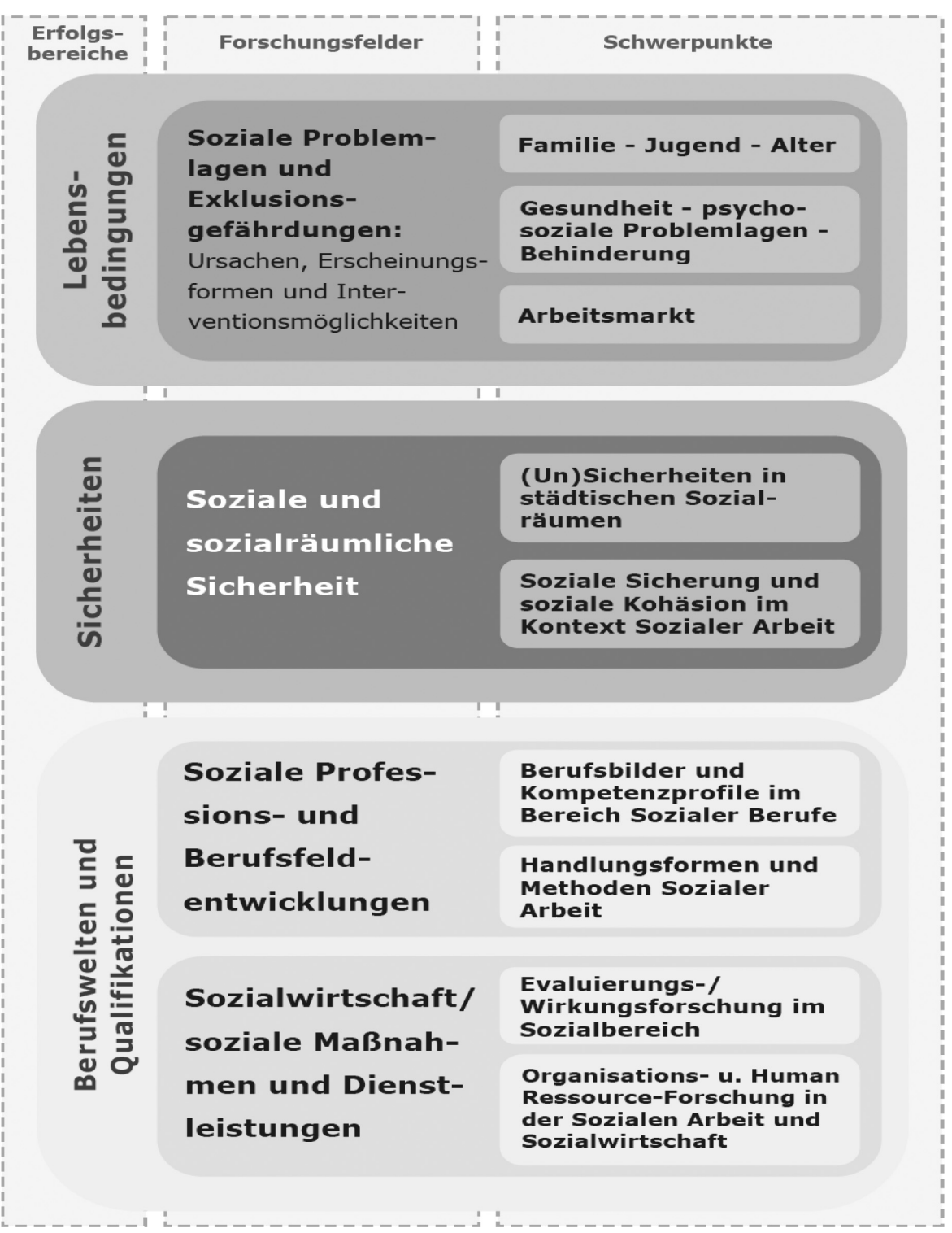

Die Forschungsfelder und Forschungsschwerpunkte des Kompetenzzentrums für Soziale Arbeit an der Fachhochschule Wien sind den drei Erfolgsbereichen »Lebensbedingungen «, »Sicherheiten « und »Berufswelten und Qualifikationen « zugeordnet.

kerinnen und Sozialpolitiker, für private wie öffentliche Einrichtungen im Sozialbereich

- Gewährleistung eines umfassenden Wissenstransfers in die Lehre als wichtigen Beitrag zur Qualitätssicherung und Weiterentwicklung der Sozialarbeitsausbildung und in die Berufspraxis, Scientific Community, Sozialwirtschaft, Politik und Gesellschaft, damit das Innovationspotenzial der Sozialarbeitsforschung gesellschaftlich nutzbar gemacht werden kann.

Die bisherigen Erfahrungen zeigen ein großes Interesse an den Forschungsergebnissen in der Sozialarbeitspraxis, Sozialwirtschaft und der Scientific Community. Beispielhaft sei hier auf die große positive Resonanz zur Studie Evaluation sozialer Interventionen verwiesen. 